\title{
Oscillations in Corn Seedling Growth as Measured by Optical Flow
}

\author{
Albert Liptay ${ }^{1}$ \\ Agriculture and Agri-Food Canada, Research Centre, Harrow, Ont. NOR 1G0, Canada \\ John L. Barron ${ }^{2}$ \\ Department of Computer Science, University of Western Ontario, London, Ont. N6A 5B7, Canada \\ Tom Jewett ${ }^{3}$ \\ Agriculture and Agri-Food Canada, Research Centre, Harrow, Ont. NOR I GO, Canada
}

Ian van Wesenbeeck ${ }^{4}$

Agriculture and Agri-food Canada, Research Centre, Harrow, Ont. NOR 1G0, Canada

Additional index words. motion analysis, leaf elongation, spectral analysis

\begin{abstract}
Growth of corn seedlings during the coleoptile stage was measured using optical flow. The measurement system was comprised of a digital camera, computer, and related software and measured growth in a continuous, noncontact manner. The use of optical flow to measure shoot elongation, i.e., image motion of the elongating seedling, was most easily computed when there were large spatiotemporal variations of the motion of the corn seedling against the background. The sensitivity of the measurement technique was in the micron per second range. Seedling growth did not occur in a smooth even manner, rather, growth was a series of varying bursts or waves of expansion that appeared to be affected by the physical growth or development of the leaves. Spectral analysis techniques were applied to extract the underlying signal from the observed time series of seedling growth rate and angle.
\end{abstract}

Physiological processes in plants have generally been reported to take date in a linear or curvilinear manner. However, it is becoming evident that, with sensitive measurement techniques (Barron and Liptay, 1994) and with sufficiently frequent sampling times (Rose and Rose, 1994), growth processes often take place in an oscillatory or periodic fashion. The oscillatory pattern is not the circadian rythm referred to by Darwin (1881), nor circumnutation necessarily, rather, the rapid fluctuations in growth that can be discerned with sensitive instruments and measurements taken with adequate frequency occur with a periodicity in the order of minutes.

The oscillatory nature of the observed seedling growth time series, as measured by optical flow in this study, is similar to, but more variables than, oscillatory behavior in plant response observed by others who measured plant transpiration (Rose et al., 1994; Rose and Rose, 1994), stomatal aperture, transpiration, and leaf water potential (Barrs, 1971). Digital signal processing techniques such as spectral analysis have been used to examine the cyclic nature of time-series data observed in nature. Spectral techniques are useful for extracting the frequency components of a signal when an underlying phenomenon is observed to be oscillatory. Spectral techniques have been used extensively to analyze time-series data in other areas of science, and Shumway (1988) includes several examples from biological and physical sciences, social sciences, and economics. Hashimoto et al. (1984) used system identification by spectral analysis to elucidate Plant responses to environmental changes in greenhouses. Their study however examined the effect of environmental factors on plant properties such as leaf temperature, $\mathrm{CO}_{2}$ concentration, and water

Received for publication 21 June 1994. Accepted for publication 13 Dec. 1994. The cost of publishing this paper was defrayed in part by the payment of page charges. Under postal regulations, this paper therefore must be hereby marked advertisement solely to indicate this fact.

${ }^{1}$ Vegetable physiologist.

${ }^{2}$ Assistant professor.

${ }^{3}$ Agricultural engineer.

${ }^{4}$ Soil physicist. content in the stem; detailed measurements of plant growth were not made. The apparent cyclic nature of the seedling growth data measured by the optical flow system used in this study lent itself to spectral analysis methods that were useful in extracting and quantifying the underlying signals that were not obvious in the raw, untransformed data.

Optical flow to measure seedling extension growth is a very sensitive, noninvasive, nondestructive measurement technique and has been documented recently (Barren and Liptay, 1994). The system measures the differences in illumination, i.e., gray levels, caused by a structure moving with respect to a stationary background. By following a sequence of changes in sequential, timelapsed images of a scene, and with appropriate mathematical filtering techniques applied to the data (Barren et al., 1994; Lucas and Kanade, 1981; Simoncelli et al., 1991), very sensitive measurement of growth can be achieved. Growth rate can be examined in very minute temporal and spatial increments because of the sensitivity of the system. Cyclic phenomena in plant growth can only be detected and uniquely determined if at least two sampling events occur within the period of the cycle; this is referred to as Shannon's sampling theorum (Phillips and Nagle, 1984; Press et al., 1986). In the past, high-frequency phenomena in plant growth may have been missed or aliased because of inadequate sampling rate.

The objectives of this study were to 1) examine the periodic extension of a corn seedling as measured by the sensitive technique of optical flow, 2) characterize the cyclical nature of growth rate data using spectral analysis techniques, and 3) demonstrate the applicability of the optical flow system to seedling growth measurements and to define some of the capabilities of the system.

\section{Materials and Methods}

Seedling growth measurement. The experimental setup was similar to that previously reported (Barren and Liptay, 1994), except that growth was monitored over a much longer time period (3 days) and during times when more rapid seedling extension was 
taking place. Corn (Zea mays) seeds were sown in modified peatmoss (ASB-Greenworld, Pointe Sapin, New Brunswick) in black polyethylene tubes $10 \mathrm{~cm}$ long and $1 \mathrm{~cm}$ id. A tube with a germinating corn seedling was inserted into a $250-\mathrm{ml}$ glass bottle containing water and coils from a water bath set at $24 \mathrm{C}$. The seedling shoot was exposed to the ambient air temperature of $22 \mathrm{C}$ \pm 1 with the roots subjected to the constant temperature of the water
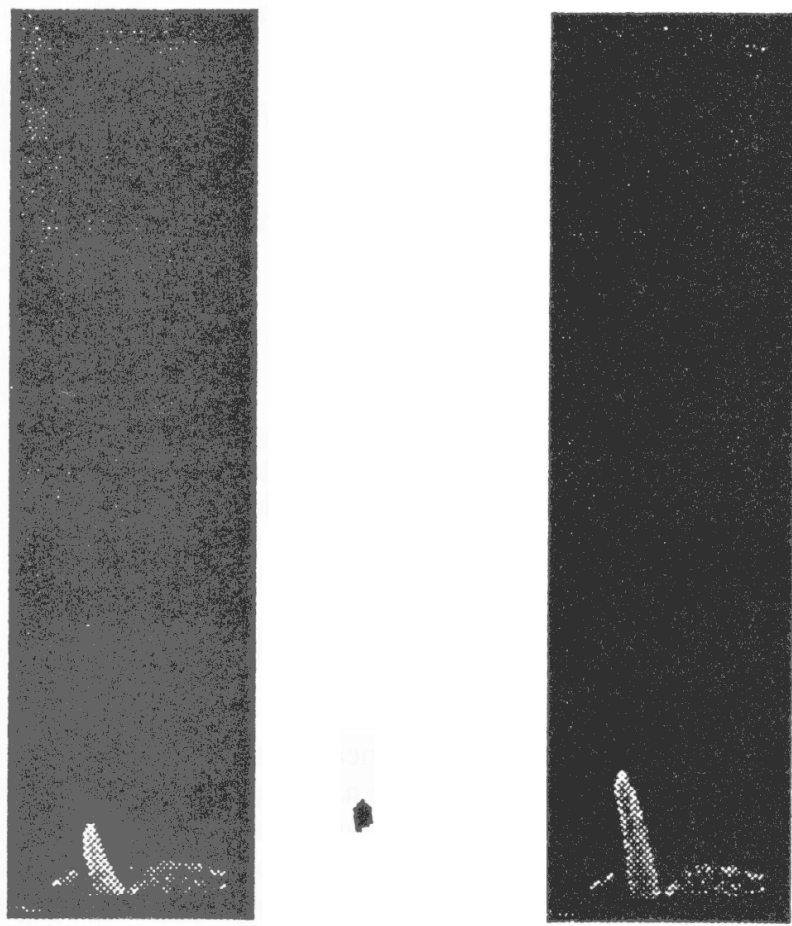

(a) Image 000

(b) Flow 000

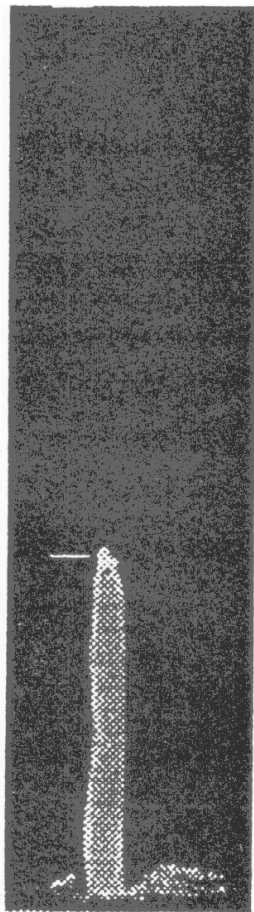

(s) Image 2,400 bath. The seedling was illuminated with a fluorescent light at an intensity of $0.7 \mu \mathrm{mol} \cdot \mathrm{m}^{-2} \cdot \mathrm{s}^{-1}$; the windows in the lab were blocked to eliminate the variability of natural light and ensure constant illumination of the seedling. A deep nap rayon velvet cloth served as the background to reduce surface light reflectivity. A CCD camera (model DXC-327; Sony) with a zoom lense (model VCL712BX; Sony) was used to image the seedlings every $2 \mathrm{~min}$; the
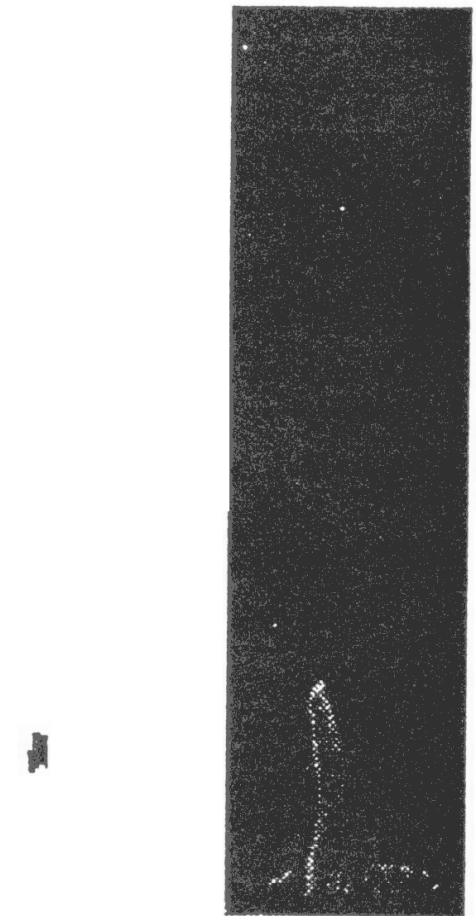

(e) Image 1,800

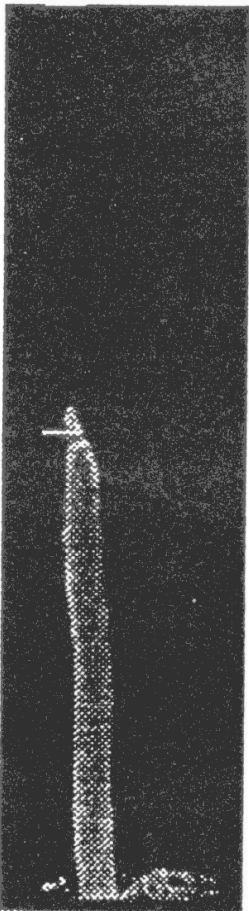

(1) Coleoptile 2,400 (j) Imege a,000 (r) Flow 1,800 (k) Lear 8,000
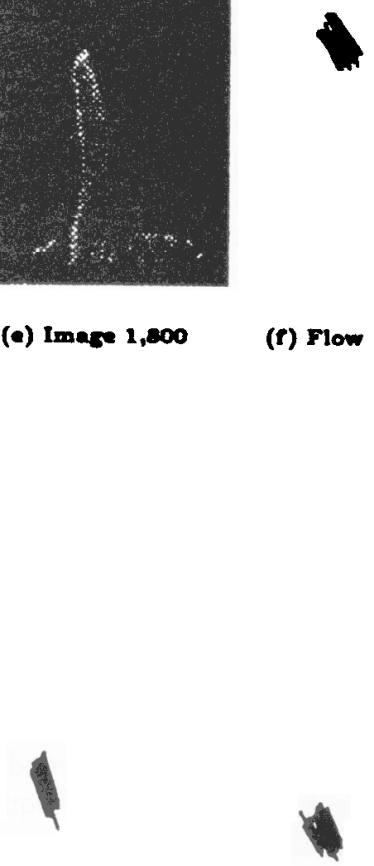

\section{1) Coleoptile 8,000}

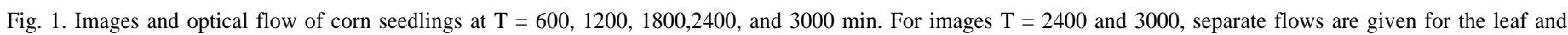
coleoptile. Flows were sampled by 2 and scaled by 100.0. A white bar indicates the manually selected leaf vs. coleoptile image flow.. 
color images were converted to gray value images using a YIQ transformation. A black and white camera would have sufficed for the measurements. The sampling rate was determined through trial and error. Computation of optical flow was done as described by Barron and Liptay (1994). The corn seedling was measured during coleoptile elongation and emergence of the first leaf through the coleoptile, i.e., over a period of $4300 \mathrm{~min}$.

Spectral statistical analysis of seedling growth. Temporal variability of the growth and angle data for the coleoptile and the leaf were analyzed using spectral statistical techniques. The power spectrum, $V_{x}^{2}\left(f_{k}\right)$, of a time series, $X_{j}$, measured over a finite time, $L$, at $N$ equal spaced time intervals $(j=1$ to $N)$ is estimated by Brillinger (1981):

$$
\begin{aligned}
& V^{2}\left(f_{k}\right)=(2 m+1)^{-1} N^{-1} \sum_{i=-m}^{m} \\
& \left\{\left[\sum_{j=1}^{N} X_{j} \cos \left(2 \pi f_{k+1} j\right)\right]^{2}\right. \\
& \left.+\left[\sum_{j=1}^{N} X_{j} \sin \left(2 \pi f_{k+1} j\right)\right]^{2}\right\}
\end{aligned}
$$

where $f_{k}=K / L, K=0,1,2, \ldots N / 2$, cycles/m, $m$ is a smoothing coefficient indicating the degree of averaging of adjacent independent frequencies and the degrees of freedom for the individual spectral variance estimates. The power spectrum proportions the variance of a variable as a function of frequency (Brillinger, 1981).

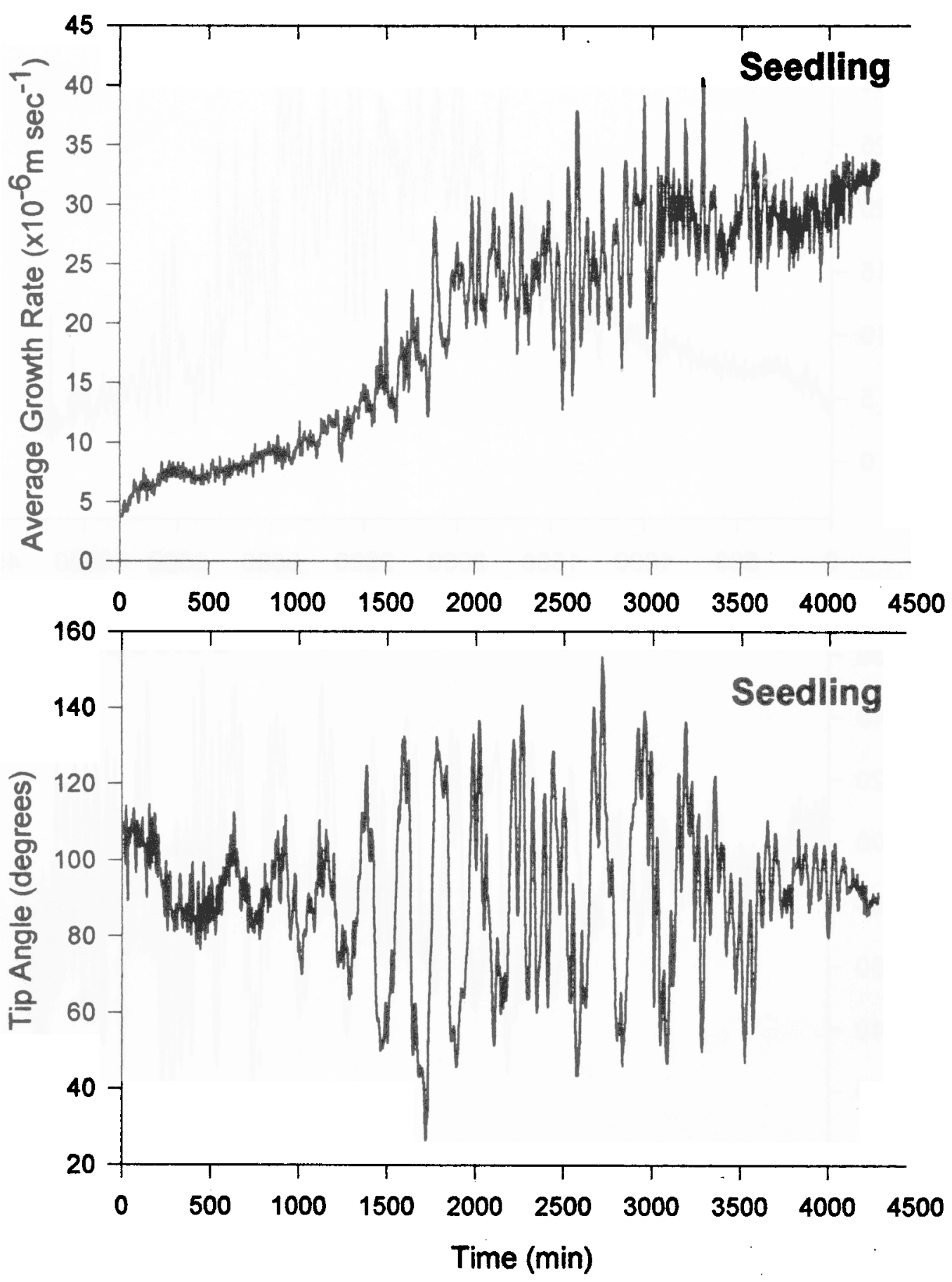

Fig. 2. Averaged growth rate and angle of growth of the seedling tip. Measurements were taken every 2 min. 
The sample cross spectrum, $V_{X I-X 2}$ between two variables is an estimate of the covariance between the two variables $X 1$ and $X 2$ as a function of frequency. The smoothed cross spectral estimate is given by Brillinger (1981):

$$
V_{X 1 X 2}\left(f_{k}\right)=\frac{1}{2 m+1} \sum_{1=-m}^{m} F_{X 1}\left(f_{k+1}\right) * F_{X 2}\left(f_{k+1}\right)
$$

where $*$ indicates complex conjugate, and

$$
\begin{aligned}
& F_{X 1}\left(f_{k}\right)=\sum_{j=0}^{N-1} X 1(j)\left[\cos \left(2 \pi f_{k} j\right)-i \sin \left(2 \pi f_{k} j\right)\right] \\
& F_{X 2}\left(f_{k}\right)=\sum_{j=0}^{N-1} X 2(j)\left[\cos \left(2 \pi f_{k} j\right)-i \sin \left(2 \pi f_{k} j\right)\right]
\end{aligned}
$$

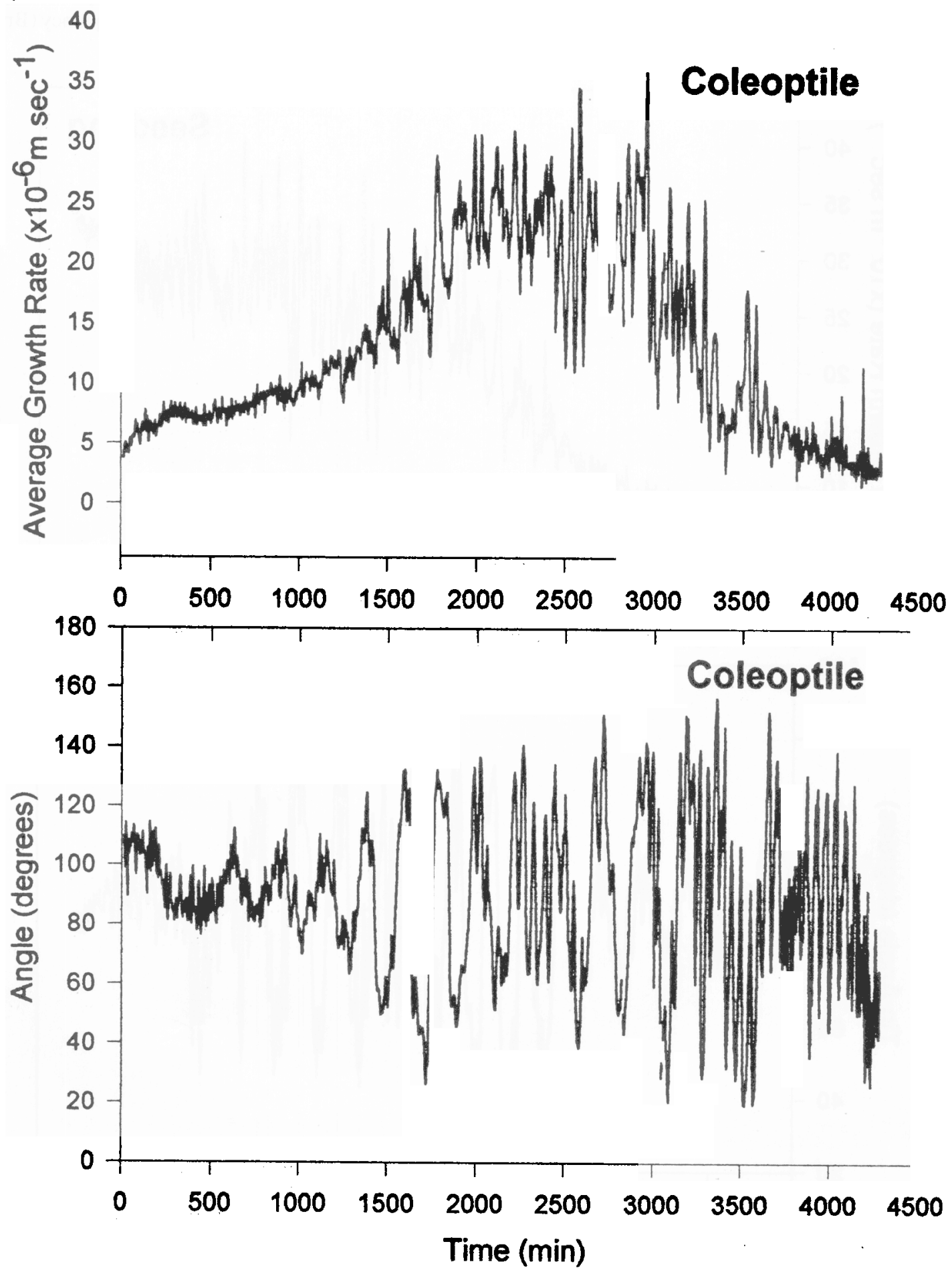

The coherency function, $R_{X I X 2}^{2}\left(f_{k}\right)$, is analgous to the coefficient of determination, $r_{X I-X X}^{2}$, and is estimated by Brillinger(1981)

[2] as

$$
R_{X 1 X 2}^{2}\left(f_{k}\right)=\frac{\left|V_{X 1 X 2}\left(f_{k}\right)\right|^{2}}{V_{X 1}^{2}\left(f_{k}\right) V_{X 2}^{2}\left(f_{k}\right)}
$$

[3] where $V_{X I X 2}\left(f_{k}\right)$ is the sample cross spectrum. 
The coherency function, $R_{X 1 x_{2}}^{2}\left(f_{k}\right)$, estimates the proportion of the temporal variance of $X 2$, which can be explained by $X 1$ as a function of frequency. The coherency function was calculated for the growth rate vs. angle data for the coleoptile and leaf tip. Confidence limits for the power spectrum and coherency spectrum were calculated according to Shumway (1986). The leaf growth rate time series was detrended by removing the best straight line linear fit to the data. All subsequent spectral analysis was conducted on the detrended series. The leaf angle data were not detrended since the data fluctuated around a mean value of $90^{\circ}$ and no linear trend was present The results of the spectral analysis were not affected by the choice of the filter window used to smooth the data. The large size of the data sequence (2400 points) minimizes the effect of series truncation in the spectral analysis.

The spectral methods were applied to quantify the frequency dependence of the variance of the time series and the correlation (coherence) of the time series. Since spectral methods distribute the variance associated with a variable as a function of frequency or period, they can be used to determine whether there are processes that are related to plant growth operating at different frequencies or periods. The tendency of the coleoptile and leaf angle to oscillate suggested that spectral analysis might be useful in extracting and quantifying the cyclic nature of the growth processes observed in this study.

\section{Results}

Sample images and their optical flow are depicted in Fig. 1. Growth rate and flow angles (Fig. 2) were averaged over each fiveimage segment (data at images $\mathrm{i}-2$ to $\mathrm{i}+2$ were averaged to produce averaged data for image i) to smooth the data and make various trends obvious. Standard deviations tended to be larger for the averaged data than for the individual measurements, but typically remained in the $3 \%$ to $5 \%$ range. Figure 3 depicts the averaged growth rate and angle data for the coleoptile from $\mathrm{T}=$
2400-4800 min. For 11 of the 950 calculations for the coleoptile, the optical flow thresholding removed all velocities in the optical flow, as they were judged to be unreliable. Averaging local growth and angle measurements ensured no nonzero values were plotted for these times.

A short segment of the leaf angle data were plotted to increase the temporal resolution indicating that in addition to the large period cycle $(\mathrm{T}=270 \mathrm{~min})$ there appear to be short periods in the leaf angle superimposed on the larger cycle (Fig. 4). The sample spectrum for leaf growth rate and angle time series indicates a large variance component for leaf growth rate at the very low-frequency range and a significant peak in the variance at a frequency of $\mathrm{f}=$ 0.035 cycles/min (Fig. 5). The variance at the very low frequency range ( $\mathrm{f}<0.01$ ) is a result of the single large cycle in leaf growth that occurs in the data series (Fig. 1). The peak in variance at the other frequency corresponds to cyclic behavior in the growth rate data with a period of $\mathrm{T}=60 \mathrm{~min}$. The sample spectrum for the angle data also indicates significant peaks in spectral variance at frequencies $\mathrm{f}=0.007$ and $\mathrm{f}=0.035$ cycles $/ \mathrm{min}$, which correspond to periods of $\mathrm{T}=250$ and $\mathrm{T}=60$ rein, respectively. This corroborates the visual observations made from Fig. 3 that there is a cycle occurring with a period of $\approx 270$ rein, and also that there is a significant cycle superimposed on this one at a higher frequency, with a period of $60 \mathrm{~min}$.

A simple correlation between the two measured time series (growth vs. angle) resulted in an $r^{2}$ value of 0.02 , indicating that the series are uncorrelated. A coherency analysis (Fig. 6) indicated that while the correlation between the two series is near 0 at low frequencies, there is a significant coherency at frequencies $>0.25$, which correspond to periods smaller than $4 \mathrm{~min}$.

\section{Discussion}

Optical flow can be used as a very sensitive, noncontact technique for measuring growth of plant structures that have a

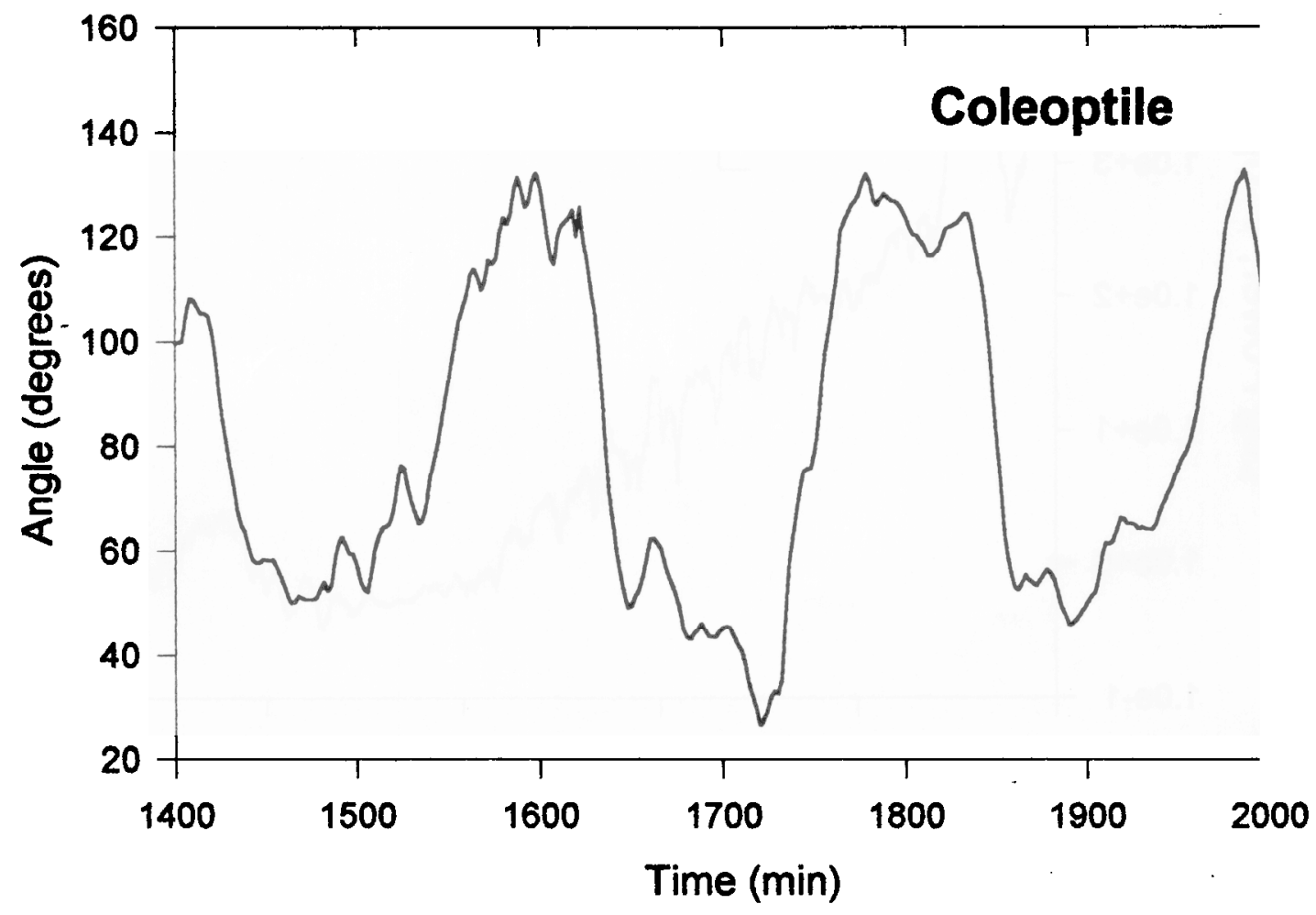

Fig. 4. Seedling angle data plotted from $T=1400$ to $T=2000 \mathrm{~min}$. Note short-wavelength cycles superimposed on larger cycles. 
relatively stable morphological structure over a brief temporal sequence. The ability of the optical flow technique to make very sensitive growth measurement very rapidly in time has enabled cyclic growth phenomena to be studied. Previously, these highfrequency cycles in plant growth could not be identified or quantified, since methods of rapidly sampling growth rate were not available.
Optical flow is not particularly suited to measurements of plants with many leaves that display nonelongating motion such as rapid leaf orientation for maximum exposure to incident radiation. It is, however, easily applicable to root tip growth analysis. Another limitation to optical flow is the measurement of stem diameter growth. Theoretically, stem width growth should be manifested as flow normal to the plant's stern, but the plant's swaying motion

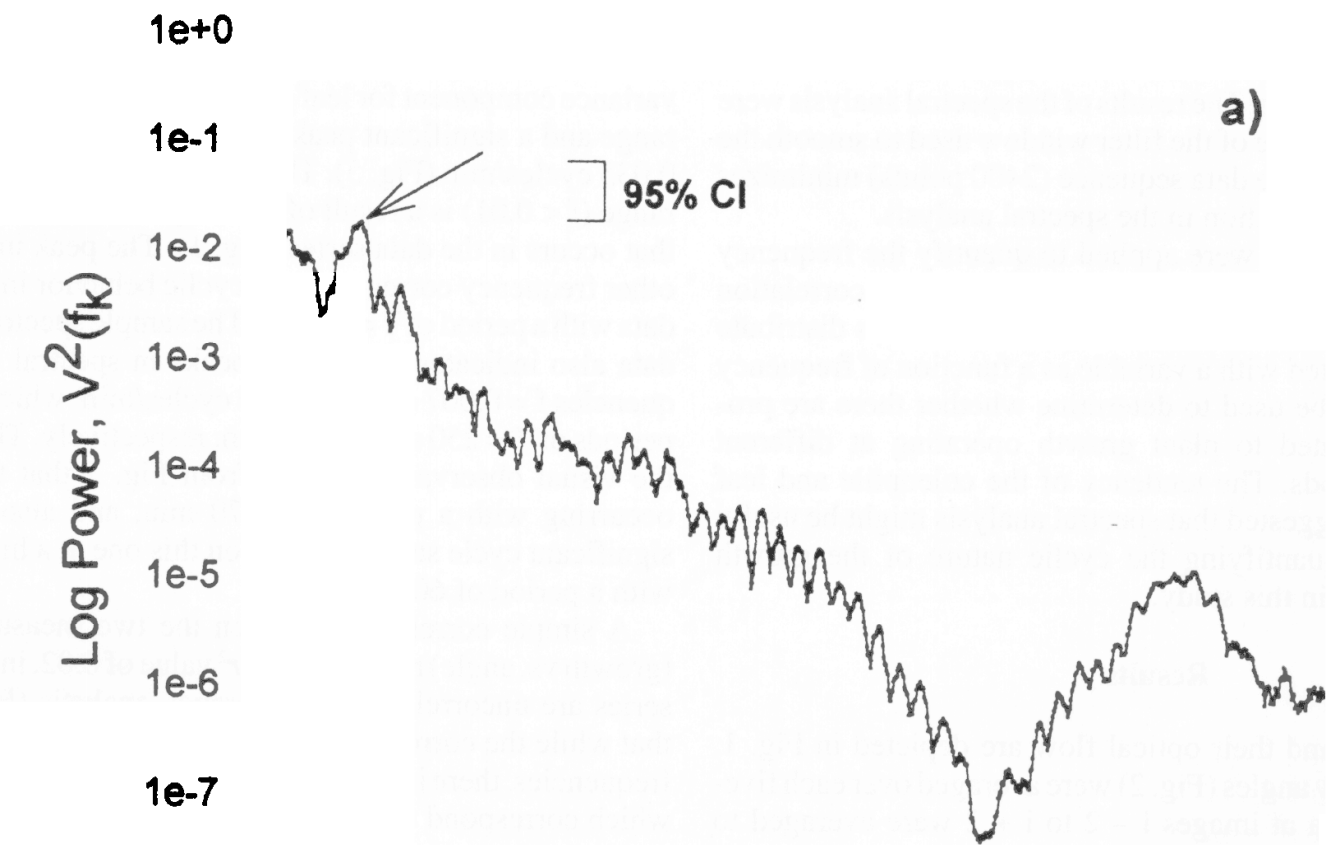

$1 e-8$

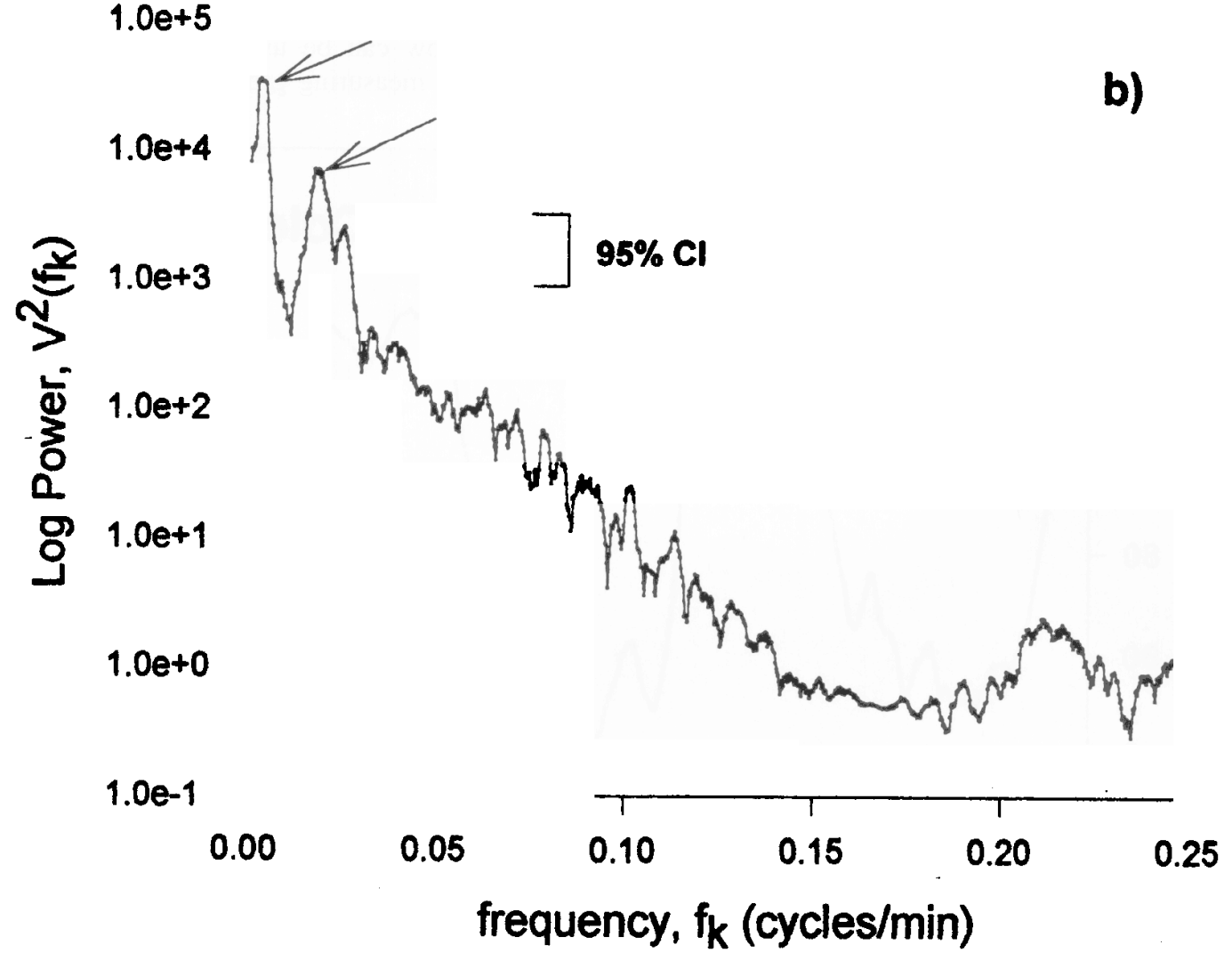

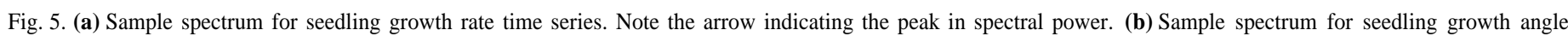
time series. Note arrows indicating peaks in spectral power at $\mathrm{f}=0.035$ and $\mathrm{f}=0.007 \mathrm{cycles} / \mathrm{rein}$. 


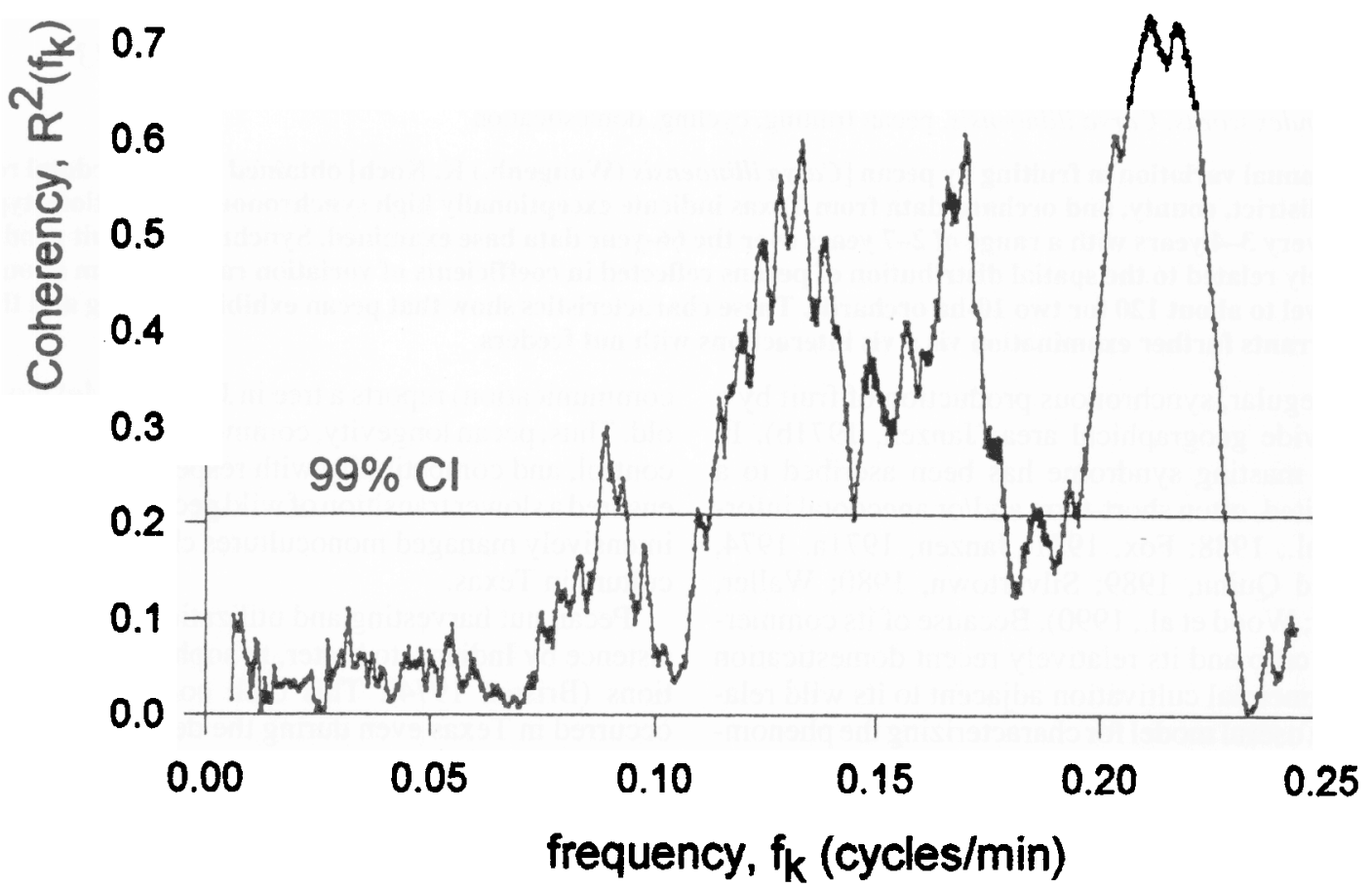

Fig. 6. Coherency spectrum between leaf tip growth rate and leaf angle.

dominated these measurements so that such data could not be retrieved with the current optical flow analysis method.

\section{Conclusion}

Spectral techniques were successfully used to extract information and quantify the nature of periodicities observed in the growth patterns of corn seedlings. The analysis also enabled the determination of the frequencies at which different growth parameters were related and, hence, will enable us, in future studies, to examine and understand the growth processes of seedlings and correlate these to morphological development of the plant. Silk (1984) reviewed the application of concepts and equations from fluid dynamics and continuum mechanics to plant developmental studies. Here, we incorporate the use of optical flow as a very sensitive growth measuring technique and apply the concepts of digital signal processing to demonstrate potential relationships to seedling growth and development. Moreover, this or another very sensitive elongation measurement technique that is able to detect oscillatory growth in plants, may have applications in confirming' other oscillatory growth phenomenon such as recently reported by Rose and Rose (1994). Future studies incorporating these and other techniques should be helpful in defining growth and development in plants more precisely.

\section{Literature Cited}

Barrs, H.D. 1971. Cyclic variations in stomatal aperture, transpiration and leaf water potential under constant environmental conditions. Annu.
Rev. Plant Physiol. 22:223-236.

Barron, J. L., D.J. Fleet and S.S. Beauchemin. 1994. Performance of optical flow techniques. Intl. J. Comp. Vis. 12:43-77.

Barron, J.L. and A. Liptay. 1994. Optical flow to measure minute increments in plant growth. Bioimaging. 2:57-61.

Brillinger, D.R. 1981. Time series: Data analysis and theory. $2 \mathrm{~d}$ ed. Holden-Day, San Fransisco.

Darwin, C. and F. Darwin. 1881. The power of movement in plants. Appleton, London.

Hashimoto, Y. B.R. Strain, T. Morimoto, and T Fukuyama. 1984. System identification of plant responses in energy conservative greenhouses. Acta Hort. 148:287-295.

Lucas, B. and T. Kanade. 1981. An iterative image registration technique with an application to stereo vision. Proc. DARPA IU Workshop. p. 121-130.

Phillips, C.L. and H.T. Nagle. 1984. Digital control systems analysis and design. Prentice-Hall, Englewood Cliffs, N.J.

Press, W. H., B.P. Flannery, S.A. Teukolsky, and W.T. Vetterling. 1986. Numerical recipes. Cambridge Univ. Press. p. 386.

Rose, M.A. and M.A. Rose. 1994. Oscillatory transpiration may complicate stomatal conductance and gas-exchange measurements. HortScience 29:693-694.

Rose, M. A., D.J. Beattie, and J.W. White. 1994. Oscillations of wholeplant transpiration in Moonlight rose. J. Amer. Soc. Hort. Sci. 119:439445.

Shumway. R.H. 1988. Applied statistical time series analysis. PrenticeHall, New Jersey.

Silk, W.K. 1984. Quantitative descriptions of development. Annu. Rev. Plant Physiol. 35:479-518.

Simoncelli, E. P., E.H. Edelson, and D.J. Heeger. 1991. Probability distributions of optical flow. IEEE Proc. CVPR, Maui. p. 3 19-315. 